\title{
STEM-LOOP 1 IN THE 5' UTR OF THE SARS CORONAVIRUS CAN SUBSTITUTE FOR ITS COUNTERPART IN MOUSE HEPATITIS VIRUS
}

\author{
Hyojeung Kang, Min Feng, Meagan E. Schroeder, David P. Giedroc, and \\ Julian L. Leibowitz*
}

\section{INTRODUCTION}

A novel coronavirus, $\mathrm{SCoV}$, was the cause of a highly lethal outbreak of respiratory disease in the winter of 2002-2003. SCoV was initially thought to represent a new coronavirus subgroup but more recent phylogenetic analyses have placed SCoV within the group 2 coronaviruses as an early split-off from the group 2 branch, which includes MHV. ${ }^{3}$ This study investigated the possibility that the SCoV UTRs, or predicted secondary structural elements within the SCoV 5' UTR, could functionally substitute for their MHV counterparts.

Consensus secondary structural models for the 5'-most 150 nts of the 5' untranslated region (UTR) of mouse hepatitis virus (MHV) and severe acute respiratory syndrome coronavirus (SCoV) were generated with ViennaRNA 1.5 and PKNOTS. The 5' UTR of both viruses is predicted to contain three major helical stem-loop structures, designated SL1, SL2, and SL4. Full-length cDNAs of the MHV genome and MHV/SCoV chimeras were assembled in vitro and transcribed to generate chimeric genomes. Replacement of the entire MHV 5' UTR with the SCoV 5' UTR resulted in nonviable genomes. In contrast, the SCoV SL1 and the SCoV 3' UTR can functionally substitute for their MHV counterparts. These chimeric viruses formed smaller plaques and grew more slowly and to lower titer than the parental MHV-A59. A59/SCoV-5, UTR/MHV-TRS and A59/SCoV-SL4-AUG chimeric viruses directed the synthesis of minus-strand genomesized RNA, but subgenomic RNAs (sgRNAs) were not made.

\section{MATERIALS AND METHODS}

The A plasmid of the MHV reverse genetic system ${ }^{5}$ was utilized as a basis for constructing a fusion of the SCoV $5^{\prime}$ UTR to the MHV gene 1 coding sequence. The

* Hyojeung Kang, Min Feng, Julian L. Leibowitz, Texas A\&M University-HSC, College Station, Texas, 77843. Meagan E. Schroeder, David Giedroc, Texas A\&M University, College Station, Texas, 77843. 
strategy employed to construct this fusion exploited "No see'm" technology to eliminate BsmBI restriction sites engineered into the ends of DNA fragments by $\mathrm{PCR}^{5} \mathrm{An}$ oligonucleotide assembly strategy was used to replace predicted stem-loop structures in MHV with their SCoV counterparts in plasmid A, ${ }^{5}$ and to replace the SCoV TRS in the MHV/SCoV-5' UTR chimera. Genome RNAs were assembled from cloned cDNAs and transcribed in vitro from the assembled cDNAs and the transcripts electroporated into BHK-R cells to recover infectious virus as described previously. ${ }^{5}$ To determine the virus specific RNAs produced by nonviable chimeras, total RNAs were extracted 8 and 24 hours post electroporation and analyzed by nested RT-PCR.

\section{RESULTS}

Consensus secondary structural models for the 5'-most $150 \mathrm{nts}$ of the 5' UTR from $\mathrm{MHV}$ and $\mathrm{SCoV}$ as well as other coronaviruses were predicted using ViennaRNA. The secondary structural models were strikingly similar, characterized by three major helical stem-loops, denoted as SL1, SL2, and SL4, respectively (Fig. 1A).

$\mathrm{MHV} / \mathrm{SCoV}$ chimeric genomes (Fig. 1B) were constructed as described in "Materials and Methods". Only cultures electroporated with A59/SCoV-3' UTR and A59/SCoV-SL1 chimeric genomes developed cytopathic effect (CPE). Virus was recovered from media, plaque purified, and expanded in DBT cells (Table 1). Average plaque sizes of A59/SCoV-SL1 and A59/SCoV-3' UTR chimeric viruses were $1.5 \mathrm{~mm}$ and $1.4 \mathrm{~mm}$ in diameter, respectively. These sizes corresponded to 54\% $(P<0.05)$ and 50\% $(P<0.05)$ of the average plaque size of MHV-A59-1000 (2.8 mm in diameter) (Table 1). The chimeric viruses achieved maximal titers approximately 50 -fold less than those achieved by wildtype MHV-A59-1000 (Fig. 2).

A

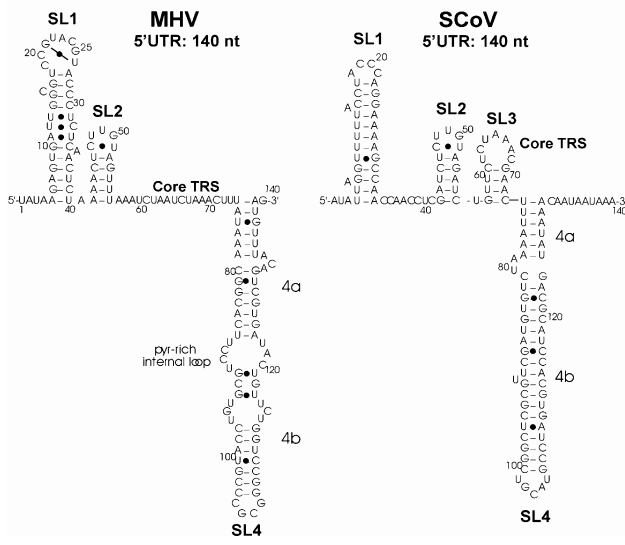

B

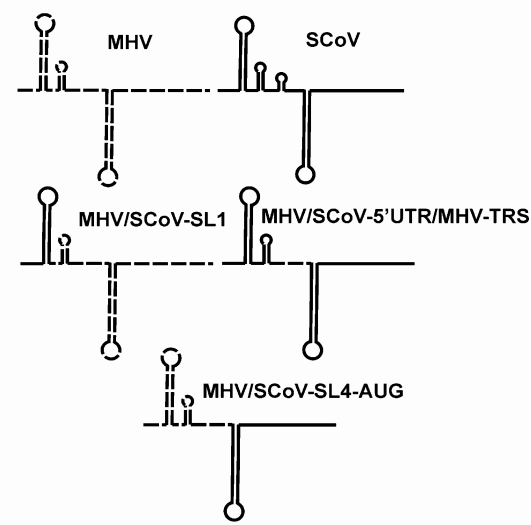

Figure 1. Predicted secondary structures of the first 140 nucleotides of MHV and SCoV 5' UTRs (A) and schematic diagram of recombinant genomes (B). 
Table 1. Characterization of MHV and SARS chimeric mutants.

\begin{tabular}{|c|c|c|c|c|}
\hline Mutant virus & $\begin{array}{c}\text { Viablilty*, } \\
\text { plaque size }(\mathrm{mm})\end{array}$ & $\begin{array}{l}\text { Minus-strand } \\
\text { genomic } \\
\text { RNA }\end{array}$ & $\begin{array}{l}\text { Minus-strand } \\
\text { subgenomic } \\
\text { RNA }\end{array}$ & $\begin{array}{l}\text { Plus-strand } \\
\text { subgenomic } \\
\text { RNA }\end{array}$ \\
\hline A59-1000 & Yes, $2.8 \pm 0.1$ & Yes & Yes & Yes \\
\hline MHV/SCOV-5' UTR & No & No & No & No \\
\hline MHV/SCOV-3' UTR & Yes, $1.4 \pm 0.1$ & $\mathrm{ND} * *$ & ND & ND \\
\hline $\begin{array}{l}\text { MHV/SCOV-5'\&3' } \\
\text { UTRs }\end{array}$ & No & ND & ND & ND \\
\hline $\begin{array}{l}\text { MHV/SCOV-5, } \\
\text { UTR/MHV-TRS }\end{array}$ & No & Yes & No & No \\
\hline MHV/SCOV-SL1 & Yes, $1.5 \pm 0.1$ & ND & ND & ND \\
\hline${ }_{\text {AUG }}^{\text {MHV/SCOV-SL4- }}$ & No & Yes & No & No \\
\hline
\end{tabular}

*Nonviable genomes failed to produce virus in three independent experiments.

** Not determined.
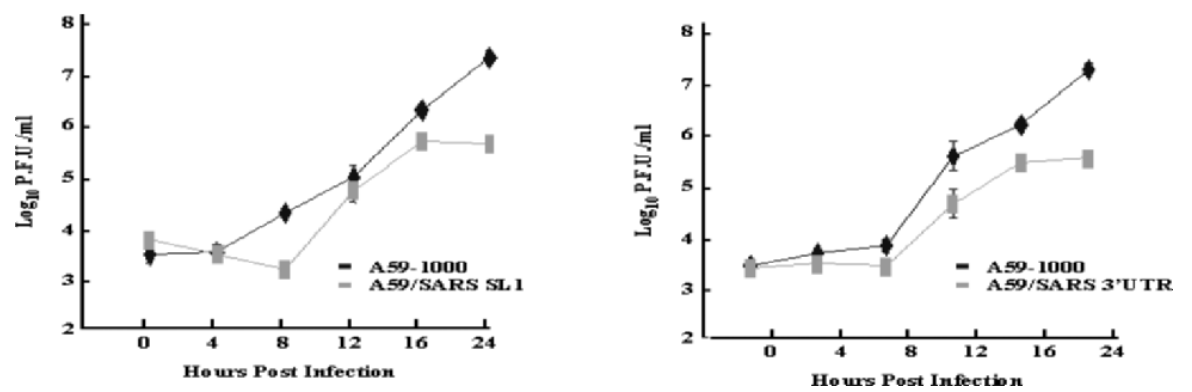

Figure 2. Growth kinetics of MHV/SCoV-SL1 and MHV/SCoV-3' UTR chimeric viruses.

Nested RT-PCR assays reveal that A59/SCoV-5' UTR/MHV-TRS and A59/SCoVSL4-AUG chimeric genomes directed the synthesis of genome-sized minus-strand RNA. In contrast, cells electroporated with A59/SCoV-5, UTR chimeric genomes failed to produce minus-strand genome-sized RNA (Table 1). We also investigated whether the nonviable chimeric genomes directed the synthesis of sgRNA7. A59/SCoV-5' UTR, A59/SCoV-5' UTR/MHV-TRS, and A59/SCoV-SL4-AUG produced neither minusstrand sgRNA7 nor plus-strand mRNA7 (Table 1).

\section{DISCUSSION}

This study showed that the entire 3' UTR and SL1 of 5' UTR of SCoV could functionally substitute for their counterparts in MHV. The functional substitution of the SCoV 3' UTR for the MHV 3' UTR is consistent with a previous study that used targeted RNA recombination to isolate a similar 3' UTR chimeric virus. ${ }^{1}$ Although the SL1s of SCoV and MHV have just $47.7 \%$ sequence identity, SL1 of SCoV is capable of forming a 
stem-loop structure similar to that of MHV SL1 (Fig. 1A). Substitution of the SCoV SL1 for the MHV SL1, which also increases the spacing between the predicted MHV SL1 and SL2 by 2 nts, did not affect viral viability, but it did decrease viral replication efficiency. These results suggest that SL1 may well play same role in replication of SCoV and MHV and supports our structural model (Fig. 1A). ${ }^{2}$ Significant sequence and/or secondary structural differences 3' to SL4 in the MHV and SCoV genomes might account for the failure to recover MHV/SCoV SL4-AUG chimeric viruses.

Failure of A59/SCoV-5' UTR and A59/SCoV-5' UTR/MHV-TRS chimeras to replicate is consistent with a previous study that reported that sequences downstream of the core TRS sequence of tranmissible gastroenteritis virus exert a strong influence on template-switching during minus-strand sgRNA synthesis. ${ }^{4}$ The three-step working model of coronavirus transcription ${ }^{6}$ postulates that the correct 5' leader TRS is required for synthesis of minus-strand sgRNA and subsequent mRNA synthesis. Our data indicate the TRS may function in the synthesis of minus strand genome RNA as well, perhaps by mediating formation of 5'-end 3'-end complex, either by RNA-RNA, protein-RNA or protein-protein interactions. Interestingly, while A59/SCoV-5, UTR/MHV-TRS and A59/SCoV-SL4-AUG chimeric viruses are capable of synthesizing genome-sized minusstrand RNA, they could not produce minus-strand sgRNAs. This suggests a defect in template switching, perhaps due to a mismatch of sequences 3' to the MHV TRS and/or improper structural presentation of the TRS in these chimeras.

\section{ACKNOWLEDGMENTS}

This work was supported by NIH grants AI051493 (J.L.L.) and AI040187 (D.P.G.).

\section{REFERENCES}

1. S. J. Goebel, J. Taylor, and P. S. Masters, The 3' cis-acting genomic replication element of the severe acute respiratory syndrome coronavirus can function in the murine coronavirus genome, J. Virol. 78, 7846-7851 (2004).

2. I. L. Hofacker, Vienna RNA secondary structure server, Nucleic Acids Res. 31, 3429-3433 (2003).

3. E. J. Snijder, P. J. Bredenbeek, J. C. Dobbe, V. Thiel, J. Ziebuhr, L. L. Poon, Y. Guan, M. Rozanov, W. J. Spaan, and A. E. Gorbalenya, Unique and conserved features of genome and proteome of SARS-coronavirus, an early split-off from the coronavirus group 2 lineage, J. Mol. Biol. 331, 991-1004 (2003).

4. I. Sola, J. L. Moreno, S. Zuñiga, S. Alonso, and L. Enjuanes, Role of nucleotides immediately flanking the transcription-regulating sequence core in coronavirus subgenomic mRNA synthesis, J. Virol. 79, 2506-2514 (2005).

5. B. Yount, M. R. Denison, S. R. Weiss, and R. S. Baric, Systematic assembly of a full-length infectious cDNA of mouse hepatitis virus strain A59, J. Virol. 76, 11065-11075 (2002).

6. S. Zuñiga, I. Sola, S. Alonso, and L. Enjuanes, Sequence motifs involved in the regulation of discontinuous coronavirus subgenomic RNA synthesis, J. Virol. 78, 980-999 (2004). 